\title{
Diagnosis for Wilson disease: this disease may not be a rare disease
}

\author{
Masaru Harada ${ }^{1}$
}

Received: 9 November 2020/Accepted: 11 November 2020/Published online: 3 January 2021

(C) Japanese Society of Gastroenterology 2021

Keywords $A T P 7 B \cdot$ Ceruloplasmin $\cdot$ Genetic disease . Wilson disease

Wilson disease is an autosomal recessive genetic disorder of abnormal copper metabolism. Until now the estimated prevalence of Wilson disease has been considered about 1 in $30,000[1,2]$. Previously, physicians sometimes disregarded this disease. Recently, many physicians recognize this disease. The diagnosis of Wilson disease is based on a combination of clinical features, serum ceruloplasmin concentration, urinary copper excretion, examination of Kayser-Fleischer ring, measurement of hepatic copper contents and genetic analysis of $A T P 7 B$ [1-3]. There are some guidelines for the diagnosis of this disease and many patients are diagnosed without genetic analysis of $A T P 7 B$ $[1,2]$. Some patients may be excluded as different hepatic diseases without genetic analysis of $A T P 7 B$.

Examination of serum ceruloplasmin is important for the diagnosis of Wilson disease. However, serum ceruloplasmin may be low in some different conditions such as heterozygotes of $A T P 7 B$ mutations, renal diseases, severe end-stage live diseases or aceruloplasminemia $[4,5]$. In addition, serum ceruloplasmin may be normal in some patients with Wilson disease [6, 7]. Measurement of hepatic copper content has been considered the best biochemical examination for the diagnosis for Wilson disease $[1,8]$. However, the distribution of copper in the liver is

Masaru Harada

msrharada@med.uoeh-u.ac.jp

1 Third Department of Internal Medicine, School of Medicine, University of Occupational and Environmental Health, 1-1 Iseigaoka, Yahatanishi-ku, Kitakyushu 807-8555, Japan sometimes inhomogeneous [1]. It is sometimes elevated in other liver diseases such as chronic cholestatic conditions [6]. Especially, some patients with idiopathic copper toxicosis were misdiagnosed as Wilson disease due to the extensively elevated hepatic copper content $[9,10]$.

In this issue of the Journal of Gastroenterology, GarciaVillarreal et al. investigated the method for the early diagnosis of Wilson disease [11]. They found a combination of measurement of serum ceruloplasmin with genetic analysis of $A T P 7 B$ was useful for the definitive diagnosis for Wilson disease. Clinical manifestations vary in patients with Wilson disease. There are hepatic, neural and other organ manifestations. Clinical features usually appear during the second or third decades. Therefore, the early diagnosis is sometimes difficult in children and patients with mild manifestations. However, the mutation of $A T P 7 B$ is present from birth in patients with Wilson disease. Considered with these problems for the diagnosis of Wilson disease, a combination of serum ceruloplasmin and genetic screening of $A T P 7 B$ is very useful, although it takes some time to obtain the result. Therefore, it may be difficult to use emergency cases. However, it must be useful for non-typical cases and asymptomatic patients. Therefore, the prevalence of this disease must be greater than the previous estimate considered from a recently published report [12]. Using this method presented by Garcia-Villarreal et al. [11] in this issue, we may be able to diagnose patients who were not diagnosed previously as Wilson disease. In additions, we will be able to eliminate the patient with idiopathic copper toxicosis who is sometimes misdiagnosed as Wilson disease [10].

Wilson disease is a rare genetic metabolic disorder that we can treat using several drugs. Therefore, a precise diagnosis is necessary using adequate methods for patients 
with this disease and this disease may not be a rare genetic metabolic disease.

\section{Compliance with ethical standards}

Conflict of interest The author declares no conflict of interest.

\section{References}

1. Roberts EA, Schilsky ML. Diagnosis and treatment of Wilson disease: an update. Hepatology. 2008;47:2089-111.

2. European Association for the Study of the Liver. EASL clinical practice guidelines: Wilson's disease. J Hepatol. 2012;56:671-85.

3. Harada M. Pathogenesis and management of Wilson disease. Hepatol Res. 2014;44:395-402.

4. Miyajima H, Nishimura Y, Mizoguchi K, et al. Familial apoceruloplasmin deficiency associated with blepharospasm and retinal degeneration. Neurology. 1987;37:761-7.

5. Walshe JM. Diagnostic significance of reduced serum ceruloplasmin concentration in neurological disease. Mov Disord. 2005;20:1658-61.
6. Martins da Costa C, Baldwin D, Portmann B, et al. Value of urinary copper excretion after penicillamine challenge in the diagnosis of Wilson's disease. Hepatology. 1992;15:609-15.

7. Gow PJ, Smallwood RA, Angus PW, et al. Diagnosis of Wilson's disease: an experience over three decades. Gut. 2000;46:415-9.

8. Merle U, Schaefer M, Ferenci P, et al. Clinical presentation, diagnosis and long-term outcome of Wilson's disease: a cohort study. Gut. 2007;56:115-20.

9. Scheinberg IH, Sternlieb I. Wilson disease and idiopathic copper toxicosis. Am J Clin Nutr. 1996;63:842S-S845.

10. Harada M, Honma Y, Yoshizumi T, et al. Idiopathic copper toxicosis: is abnormal copper metabolism a primary cause of this disease? Med Mol Morphol. 2020;53:50-5.

11. Garcia-Villarreal L, Hemandez-Ortega A, Sanchez-Monteagudo A, et al. Wilson disease: revision of diagnostic criteria in a clinical series with great genetic homogeneity. J Gastroenterol. (in press).

12. Gao J, Barackley S, Mann JP. The global prevalence of Wilson disease from next-generation sequencing data. Genet Med. 2019;21:1155-63.

Publisher's Note Springer Nature remains neutral with regard to jurisdictional claims in published maps and institutional affiliations. 